\title{
White-light speckle image correlation applied to large-strain material characterization
}

\author{
Giovanni B. Broggiato — Luca Cortese \\ University of Rome "La Sapienza" \\ Mechanics and Aeronautics Department \\ via Eudossiana, 18 - 00184 Rome (Italy) \\ giovanni.broggiato@uniroma1.it \\ luca.cortese@uniroma1.it
}

\begin{abstract}
In experimental mechanics, the possibility of tracking on component surfaces the full-field stress and strain states during deformation can be utilized for many purposes such as formability limits determination, quantification of stress intensification factors, material characterization and so on. Concerning the last topic, an interesting application could be a direct identification of the elasto-plastic material response up to large deformation. It is well known, in fact, that with traditional measurement devices it is possible to retrieve the true equivalent stress versus true equivalent strain data from tensile tests only up to the onset of necking, where localization starts to occur. This work aims to show how from the knowledge of a tensile test full-field strain and of load data it will be possible to obtain the full-stress field as well as the complete material elasto-plastic behavior.

RÉSUMÉ. En mécanique expérimentale, la possibilité d'avoir accès à des champs de contrainte et de déformation à la surface d'éprouvettes peut être utilisée de différentes manières telles que la détermination de courbes limites de formage, la quantification de facteurs d'intensité des contraintes, la caractérisation de matériaux, etc. En ce qui concerne ce dernier point, une application intéressante concerne l'identification directe de la réponse élasto-plastique de matériaux jusqu'à de forts niveaux de déformation. Il est bien connu qu'avec des moyens de mesure traditionnels, il est possible de déterminer la contrainte équivalente vraie en fonction de la déformation équivalente vraie à partir d'un essai de traction jusqu'à l'apparition de la striction, pour laquelle on observe une localisation de la déformation. Cet article a pour but de montrer qu'à partir de champs de déformation et de la mesure de la force appliquée, on peut obtenir le champ de contrainte ainsi que le comportement élasto-plastique complet du matériau.

KEYWORDS: image correlation, full-field strain measurement, plasticity, large deformation, inverse methods.

MOTS-CLÉS: corrélation d'images, mesure de champs de déformation, plasticité, grandes déformations, méthodes inverses.
\end{abstract}

DOI:10.3166/EJCM.18.377-392 @ 2009 Lavoisier, Paris

EJCM - 18/2009. Pictures and finite elements, pages 377 to 392 


\section{Introduction}

Dealing with processes where large deformations are involved many modeling problems may arise. Using FE codes, there is the necessity to put particular care in all the phases needed to set-up the model equivalent to the process: element type formulation, material model choice, discretization strategy, exact application of boundary conditions and loads. Then, the correct options regarding plasticity theories, tensor formulation, taking into account large displacements and large strains effects and time step value must be well weighed up in order to obtain convergence and accuracy of the non-linear solution. Almost all of these choices are specific competences of any FEM user and are beyond the scope of this work.

Nevertheless, aside from one's modeling strategy, a correct description of the elastic-plastic constitutive relation up to high strains is always needed. It is also clear that the correctness of any numerical simulation at large strain depends on the fidelity of the chosen material model with respect to its real behavior.

Hereafter, the study will be limited to isotropic material subjected to monotonic loading conditions. True-stress vs. true-strain curve must be provided to FE code in order to describe the correct elasto-plastic behavior. Apart from the analytical expression of the adopted model, the problem is to retrieve enough information from experimental data for calibration purposes. For an isotropic model, simple tensile tests are sufficient to identify the material constants. The main difficulties lie in the lack of useful stress-strain data in the post-necking regime.

The data returned by a clip gage are only correct up to the onset of necking: after that, in fact, the stress state becomes triaxial. Thus a different strain measurement device is needed to retrieve useful data directly from the test at high deformations.

A first answer to this problem is based on the use of Bridgman's formulas (Bridgman, 1952, McClintock, 1966). Bridgman's method is founded on an analytical description of the stress state in the minimum section of cylindrical specimen in the post-necking regime. The evolution of three quantities must be measured during deformation as shown in Figure 1a: load, minimum diameter and radius of curvature of the necking section.

One major advantage of the Bridgman's method is that parameter estimation is not required. Once load, minimum neck diameter and radius of curvature during deformation are available the entire post-necking stress-strain curve can be estimated. As an example, in Figure $1 \mathrm{~b}, \sigma-\varepsilon$ curves for a typical stainless steel are shown. Tests have been performed on smooth cylinder specimens with the aid of the optical equipment to acquire specimen silhouette during plastic deformation. Load data come directly from load-cell, while, by means of a post-processing software, the necessary geometric data can be retrieved from the images grabbed and stored during the test. A comparison between clip gage values, mean axial stress-strain values and Bridgman's corrected values is put forth. Clip-gage response fails after the onset of necking while 
the difference between the approximated mean axial stress-strain data and the Bridgman's correction is appreciable in the post-necking regime.

A second different approach to describe the post-necking behavior is derivable from the use of inverse methods (Ling, 1996).

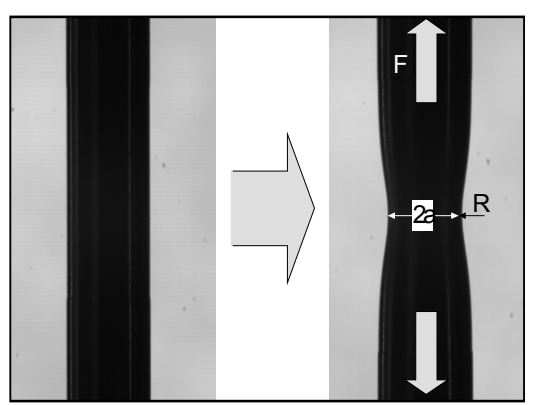

(a)

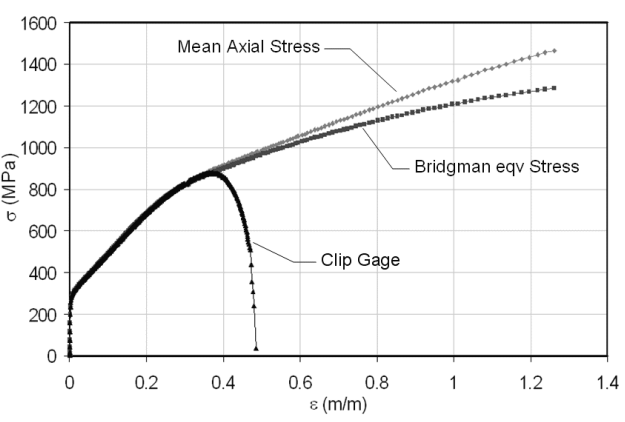

(b)

Figure 1. Bridgman's method, acquired data and comparison between clip-gage data, mean axial stress-strain data, corrected data up to high deformation

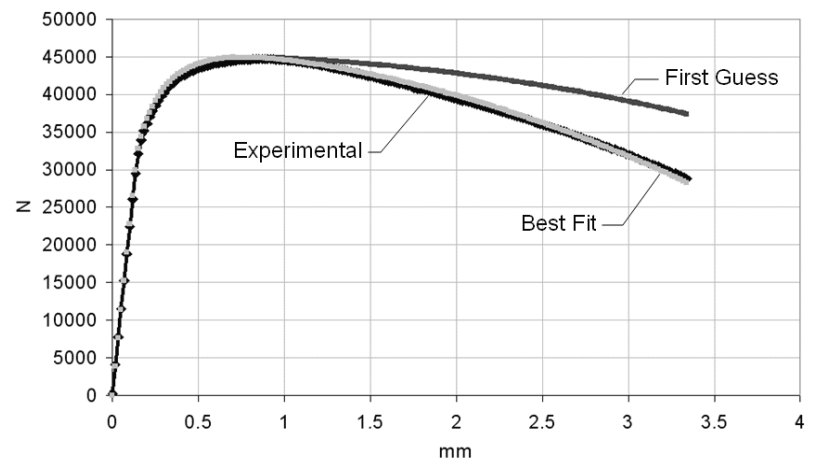

Figure 2. Ling's extrapolation: load-displacement best fit results on smooth cylinder specimens for a quenched and relieved steel for cold working applications

Suppose to write a weighted linear combination of the linear tangent extrapolation and of the power law extrapolation of the experimental data till onset necking, and to use this $\sigma-\varepsilon$ curve to perform a numerical simulation of the tensile test. Minimizing the difference between the load-displacement curve coming from the test with the corresponding FEM model response, the weight value of the linear combination can be achieved in a few iterations with a fairly good accuracy (see Figure 2). 
The first method allows stress-strain determination from direct measures, but requires additional experimental equipment and a rather complex post-processing phase. Furthermore, some simplifying hypotheses in the analytical formulation of the stress state affect the result accuracy.

The latter method instead (Ling, 1996), is more accurate in its prediction, and is more popular than the previous. The major disadvantages, reside in the complexity of the inverse procedure to be coded for fine tuning and in the additional effort due to the interaction with a FE code. Another key drawback is that local stress-strain behavior is retrieved from global data; doubts may arise that the information provided is sufficient for a unique characterization especially when the model depends on more than one parameter.

Recent works instead (Cooreman, 2007, Grediac, 2002), have put forth how inverse methods could be applied to identify material parameters exploiting local data. In general, local information is gathered from experimental tests using digital image correlation analysis and later post-processed through different techniques. The works differ on the way the collected data are used; most of them minimize the difference between measured and estimated strain values. In any case, the advantage of local data availability is that much more information can be utilized for calibration purposes. Following the trend of these recent works, a procedure is proposed here, instead of use a stress-based approach, in order to identify material constants from local data. The main goals are:

- obtaining stress-strain constitutive data directly from experiments, without any FEM support;

- exploiting the higher amount of information possible, not discarding global quantities, but in addition relying on local data whenever possible.

The procedure can be summarized as follows. At first, from a tensile test on flat specimens cut from the material under investigation, the full strain field is retrieved up to large deformation. This data are obtained by processing the sequence of digital images jointly and synchronously acquired along with the load during the uniaxial test. The local deformation field is then computed exploiting the white-light speckle image correlation technique (Broggiato, 2004, 2008) that will be explained in the next section.

Once the strain field is available, it is possible to proceed in a FEM like fashion, using the equations governing the plastic flow to get the local stress field for the tested specimen. This task is quite simple assuming the knowledge of the complete stress-strain relation. But, also without this information, using the collected global experimental load data, it is achievable to obtain stress field and material constitutive relation concurrently. This is made possible providing a parameter dependent analytical expression for the uniaxial stress-strain curve, retrieving the unknown parameter dependent stress field and finally imposing global equilibrium conditions on properly chosen specimen cross sections. On each section axial force is computed summing up all local axial stress components. 
An optimization algorithm handles parameter changes until a complete matching between computed and experimental axial load curves is reached, for all the sections simultaneously. Best fit parameters are returned. In this way all local information is utilized for material tuning purposed. It is important to underline how, for the method to work, the hypothesis of plane stress condition must be strictly obeyed. Only in this case, in fact, the retrievable surface stress state is representative of the whole field inside the material. For this reason, flat specimen geometry has been chosen, in order to maintain the plane stress conditions up to the higher deformation as possible.

\section{Digital image processing technique}

The strain measurement techniques based on image correlation, that were born a couple of decades ago (Sutton, 1983, Bruck, 1989, Cheng, 2002, Amodio, 2003, Besnard, 2006, Robert, 2007), have recently become quite popular because of the continuous improvement of CCD and CMOS sensors in photometric quality, resolution and speed, and for the contemporary availability of low cost computers able to process hundreds of pictures in a few minutes. These aspects are particularly well suited to face full-field measurement, where a large amount of data should be extracted from each image. In full-field strain measurement, the use of a "global approach" for image correlation is advisable. This method constrains the displacement field to be continuous across the entire image, so that the description of material behavior results more reliable (Broggiato, 2004). In fact, the image field is divided into sub-images in the same way as the element mesh is built for a finite element analysis. During the correlation computation, the elements on the deformed image cannot freely change their shape to match the elements on the undeformed image, but it is required that their displacements accomplish the congruency constraint caused by the shape functions that describe the deformation of each image element.

Considering the correlation between two images, the evaluation of the displacements is carried out as follows: the undeformed picture is divided in a fixed and regular grid of square elements; the portion of the image that is framed by the four corner nodes of each element is associated to them. On the deformed image a similar operation is performed, but here the node grid is neither fixed nor regular.

Consequently, to associate a square sub-image to each element of the deformed grid, their content has to be remapped into sub-images equal in size and shape to the undeformed ones. To perform the remapping operation, the deformed image is resampled on a distorted pixel grid: a bilinear shape function is used to locate the new sampling points and a bicubic interpolation scheme is adopted to estimate the gray level of the resampled pixels.

The target of the global correlation algorithm is to arrange the nodes of the deformed grid so that the sub-images coming from the resampling operation look (in speckle distribution) as close as possible to the corresponding sub-images of the reference image. 
This problem is solved minimizing (by the nonlinear least squares method) the difference between the element sub-images in the two configurations and using the deformed grid node coordinates as unknowns. At convergence, the displacement field is completely determined, thus at each node the strain components can be evaluated by means of the Cauchy-Green theory for large strain description (Amodio, 1995).

A further development of the global approach in image correlation is the multiimage correlation method. When a single tensile test is monitored collecting some hundreds of images, the spatial constraint, given by the global approach, can be extended to time improving the smoothness of displacement evolution and, consequently, the accuracy of measured data. Doing so, the shape function used to locate the new sampling points on deformed configuration becomes a function of both space and time that involves a set of typically 5 or 7 consecutive images in each correlation step.

This method, originally introduced to measure the strain-rate field (Broggiato, 2004), allows to improve the outcome accuracy of every numerical procedure that is not based on total strains but on strain component increments as require here (see next section, Equation [5]). From this point of view, the strain-rate and the stress components computations closely resemble each other. Additional details about this method can be found in (Broggiato, 2004).

\section{Large-strain material characterization procedure}

As a first step, the procedure to obtain the stress field starting from the strains measured on a thin sheet will be illustrated. It must be again underlined that plane stress condition is required. Specimen geometry has to be devised to ensure this hypothesis. Then, providing the true stress - true strain material response and the full-field strain evolution (which is available for the surface of the specimen only, but that in plane stress condition is also representative of the in-core stress state), the following Prandtl-Reuss formulas (Khan, 1995, Chung, 1988) provide the relation between deformation and tension. Here they are expressed in a general rate form, which allow full-stress field updating:

$$
\dot{\varepsilon}_{i j}^{\prime}=\dot{\varepsilon}_{i j}^{\prime e l}+\dot{\varepsilon}_{i j}^{\prime p l}=\frac{1}{2 G} \dot{\sigma}_{i j}^{\prime}+\dot{\lambda} \sigma_{i j}^{\prime}
$$

where $\varepsilon_{i j}{ }^{e l}$ and $\varepsilon_{i j}{ }^{p l}$ are the elastic and plastic strain tensors, $G$ the shear modulus and $\sigma_{i j}$ the deviatoric stress tensor. The proportionality factor $\dot{\lambda}$ is a first order homogeneous function of the strain rate. Assuming von Mises yield criterion it can be expressed as:

$$
\dot{\lambda}=\frac{3 \dot{\varepsilon}_{e q}^{p l}}{2 \sigma_{Y}}
$$


where $\sigma_{Y}=\sigma_{Y}\left(\varepsilon_{e q}^{p l}\right)$ represents the current yield stress and $\varepsilon_{e q}^{p l}$ the total equivalent plastic strain.

Since $\dot{\varepsilon}_{k k}^{p l}=0$ the volume change is governed by the elastic deformation only:

$$
\dot{\varepsilon}_{k k}^{e l}=\frac{\dot{\sigma}_{H}}{K}
$$

Being $\sigma_{H}$ the hydrostatic stress and $K$ the bulk modulus. Dealing with large strains elastic shape and volume changes can be neglected so that [1] and [2] reduce to:

$$
\begin{gathered}
d \varepsilon_{x x}^{p l}=\frac{d \varepsilon_{e q}^{p l}}{\sigma_{Y}}\left[\sigma_{x x}-\frac{1}{2}\left(\sigma_{y y}+\sigma_{z z}\right)\right] \\
d \varepsilon_{y y}^{p l}=\frac{d \varepsilon_{e q}^{p l}}{\sigma_{Y}}\left[\sigma_{y y}-\frac{1}{2}\left(\sigma_{z z}+\sigma_{x x}\right)\right] \\
d \varepsilon_{z z}^{p l}=\frac{d \varepsilon_{e q}^{p l}}{\sigma_{Y}}\left[\sigma_{z z}-\frac{1}{2}\left(\sigma_{x x}+\sigma_{y y}\right)\right] \\
d \varepsilon_{x y}^{p l}=\frac{3 d \varepsilon_{e q}^{p l}}{2 \sigma_{Y}} \sigma_{x y} \\
d \varepsilon_{y z}^{p l}=\frac{3 d \varepsilon_{e q}^{p l}}{2 \sigma_{Y}} \sigma_{y z} \\
d \varepsilon_{z x}^{p l}=\frac{3 d \varepsilon_{e q}^{p l}}{2 \sigma_{Y}} \sigma_{z x}
\end{gathered}
$$

Prandtl-Reuss equations have been intensively validated experimentally. The approximation is acceptable for almost all engineering applications.

For plane stress conditions the above expressions can be specialized and easily inverted to provide stresses given the plastic deformation tensor:

$$
\begin{aligned}
& \sigma_{x x}=\frac{\sigma_{Y}}{d \varepsilon_{e q}} \frac{d \varepsilon_{x x}+\frac{1}{2} d \varepsilon_{y y}}{1-v_{p}^{2}} \\
& \sigma_{y y}=\frac{\sigma_{Y}}{d \varepsilon_{e q}} \frac{d \varepsilon_{y y}+\frac{1}{2} d \varepsilon_{x x}}{1-v_{p}^{2}} \\
& \sigma_{x y}=\frac{2}{3} \frac{\sigma_{Y}}{d \varepsilon_{e q}} d \varepsilon_{x y}
\end{aligned}
$$


where $v_{p}$ is the Poisson's ratio in the plastic regime. This last operation does not take much computing time so that, if strain information is available in real-time, stress determination could be available in real-time as well.

One computational disadvantage of a direct use of plastic flow equations is that stress state should be locally inquired to check if it falls inside or outside the previous yielding surface, so that elastic or plastic equations could be chosen accordingly.

A valid alternative to overcome this drawback is to proceed relying on the radial return mapping algorithm. This procedure, which is the standard choice in FE codes, is quite simple: firstly the stress is updated assuming that the response is elastic and then, if it is outside the yield surface, the stress is projected back on to the closest point of the yield surface. Of course for a strain hardening material the yield surface is not constant, but expands during the plastic flow, so that the stress is to be projected on the updated current surface. The data available at the beginning of each radial return update are the previous stress, the equivalent plastic strain, and the strain tensor increment in the time-step. As output, the radial return procedure updates stress tensor and equivalent plastic strain at the end of the time-step itself. By this algorithm, both elastic and plastic domain can be handled automatically and elastic effects in the plastic region can be accounted for. In this works, the same level of accuracy of results have been found using both methods described above.

Anyway, once the method for getting stress field from strains can be regarded as consolidated, let us now see how, if the strain field is acquired synchronously with global tensile load data, equivalent stress vs. equivalent plastic strain curve can be identified along with stress field. The constitutive curve has been modeled analytically as:

$$
\sigma=\sigma_{0}+A\left(1-e^{-B \varepsilon_{p}}\right)+C \varepsilon_{p}+D \varepsilon_{p}^{2}
$$

$\sigma_{0}$ is the initial yield stress, the second term models the initial plastic zone while the last ones the large deformation region which for most materials is quite straight or slightly curved. The unknown parameters are $A, B, C$ and $D$. For many materials $D$ can be disregarded.

Then, for some arbitrary specimen cross sections, local axial stresses are summed up to give global axial load. An error function, intended as the difference between experimental and (parameter dependent) computed global load data can be minimized by means of an optimization algorithm. Minimization is run on all deformation steps available simultaneously in order to exploit the maximum information possible. The outcome of the procedure is the unknown best fit parameter set. Doing so, the material constitutive law and stress-field can be concurrently built up. 

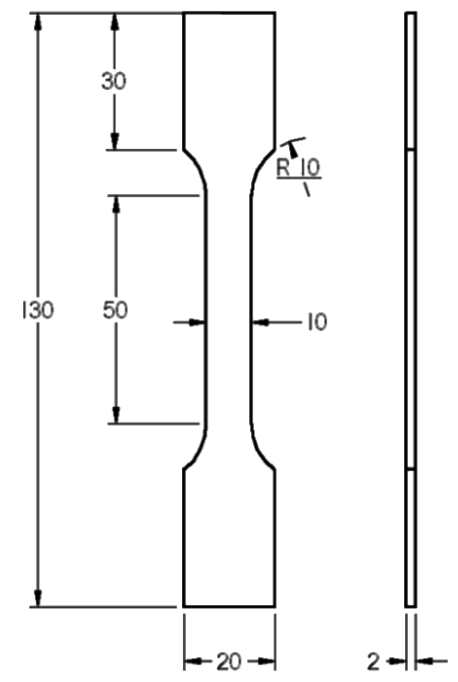

Figure 3. Detail of the flat specimen used for material characterization

A step by step description of the whole calibration process is now put forth:

1. From speckle experimental data, grid node coordinates, connectivity and strain field are retrieved for each deformation step. Corresponding global data, load and displacement (from clip gage) are collected too.

2. For each node, the appertaining area of the part of the continuum it represents is estimated. Then, area projection along the perpendicular to the axial loading direction is computed as well.

3. Specimen cross-sections where plastic behavior is exhibited in greater extent are selected. Nodes lying on these sections (on the undeformed grid) are reorganized in subsets. The section evolution during all steps of the deformation is followed.

4. First guess parameters for constitutive curve expressed by [6] are chosen. Here the choice is not a serious issue, since a good first attempt could be provided from the stress-strain curve, valid up to the onset of necking, easily determined from loaddisplacement acquired data. No particular difficulty has been encountered anyway when starting from parameters retrieved from literature constitutive curves for the specific material. Given that, by Prandtl-Reuss equations or radial return, the subsequent first guess stress field is computed.

5. From the knowledge of the stress field for each previously located section the global axial load is retrieved as:

$$
F_{a}=\sum_{i} \sigma_{i}^{a} A_{i}^{p}
$$


where $\sigma_{i}^{a}$ is the local axial component of stress, $A_{i}^{p}$ is the previously determined projection of the area appertaining to the $i$-th node where the index $i$ sweeps all the nodes of the section. Of course $F_{a}$ is calculated for all the selected sections as well as for all the available time increments.

6. An error vector is built up, expressed as the difference, section by section, and increment by increment, of the calculated and experimental axial load. A scalar error function is obtained evaluating the L2 norm of this error vector.

7. Through an iterative procedure, an optimization algorithm varies the unknown parameters of the constitutive law until the error function is minimized. Here both zero order simplex method and first order non-linear least squares algorithms have been tested with success. The output of this phase is the optimum parameter set that provides the correct stress-strain relation up to large deformation as well as the final stress field in the material.

\section{Experimental tests and main results}

The experimental setup consists of a $250 \mathrm{kN}$ servo-hydraulic MTS axial machine capable to perform tension tests on flat specimens as shown in Figure 3. The material under investigation is a high strength steel for thick tube applications. Axial load and deformation are acquired by a load-cell and a clip gage. The optical equipment is mounted on a frame clamped on a column of the testing machine (see Figure 4). The core element consists in a high-resolution $(3000 \times 2200$ pixel) Pixelink A781 CMOS camera for image acquisition. It can be actuated vertically to follow specimen elongation to guarantee the image to be always centered within the region of interest. Optical and traditional data acquisitions are obviously synchronized. Tests are performed at a quasi-static constant speed controlling MTS actuator displacement. All tests ended at specimen failure. Snapshots of the specimen surface are collected at sampling frequency of $0.5 \mathrm{~Hz}$. All other data are sampled at $50 \mathrm{~Hz}$.

To process the strain field, the specimen area framed in each image is divided in a $12 \times 24$ element grid. The dimension of each element is $128 \times 128$ pixels. Thus the displacement components are computed in 325 nodes. Typically, calculations last less than 2 seconds per image.

Figure 5 shows acquired optical data at various levels of deformation. In $5 \mathrm{a}$ the image is taken at small constant strain (equivalent true plastic strain: $\varepsilon=0.02 \mathrm{~m} / \mathrm{m}$ ) before the onset of necking. In $5 \mathrm{~b}$ strain localization has already started $\left(\varepsilon_{\max }=0.4\right.$ $\mathrm{m} / \mathrm{m})$ while in $5 \mathrm{c}$ specimen is undergoing large strain and it is close to failure $\left(\varepsilon_{\max }=\right.$ $0.7 \mathrm{~m} / \mathrm{m})$.

In Figure 6 instead, the computed strains are represented. Overlapping of results on the correspondent raw image allows the immediate visualization of the local fullstrain field. The three images of Figure 6 are caught at same strain levels of Figure 5 . 


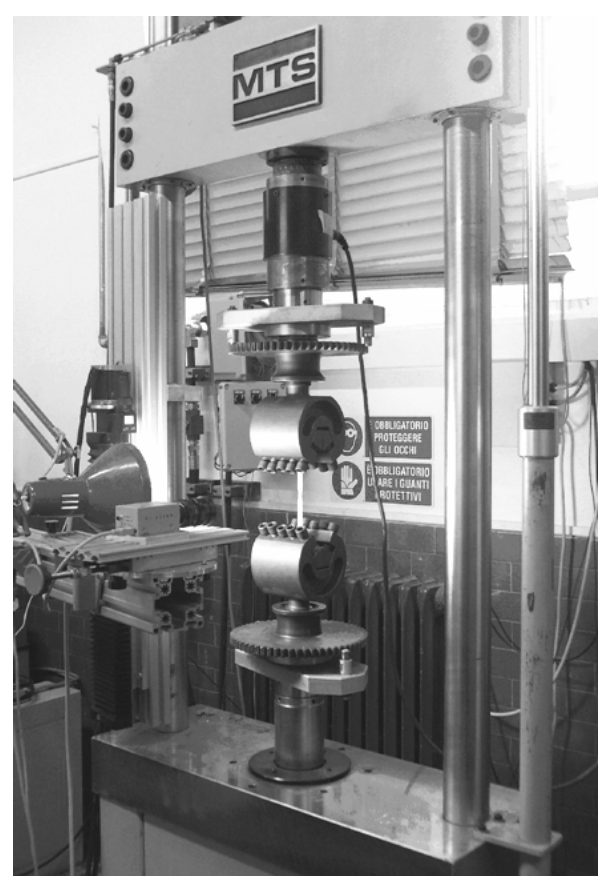

Figure 4. Optical acquisition experimental equipment

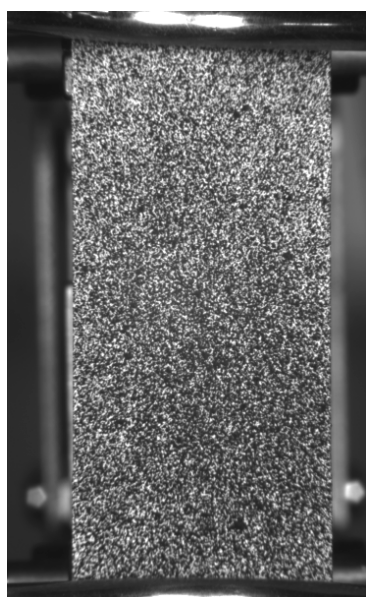

(a)

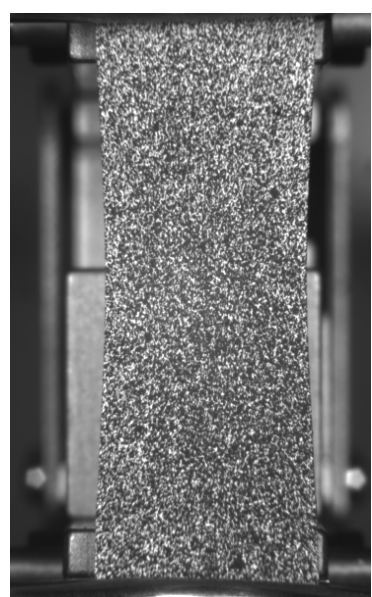

(b)

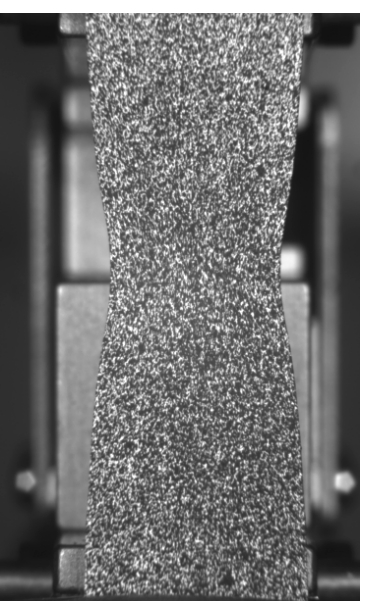

(c)

Figure 5. Acquired optical data during test: a) pre-necking, small strain. b) onset of necking, strain localization. c) post-necking, large deformation 


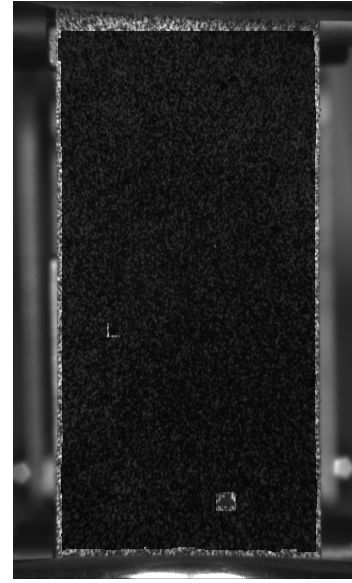

(a)

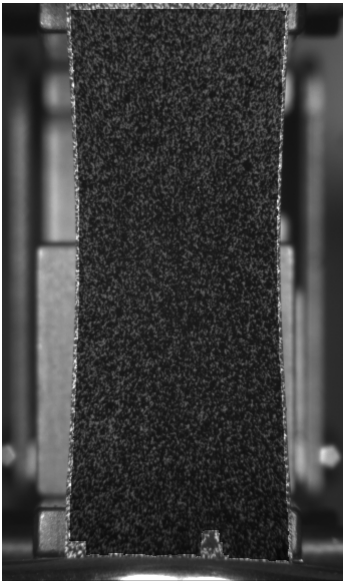

(b)

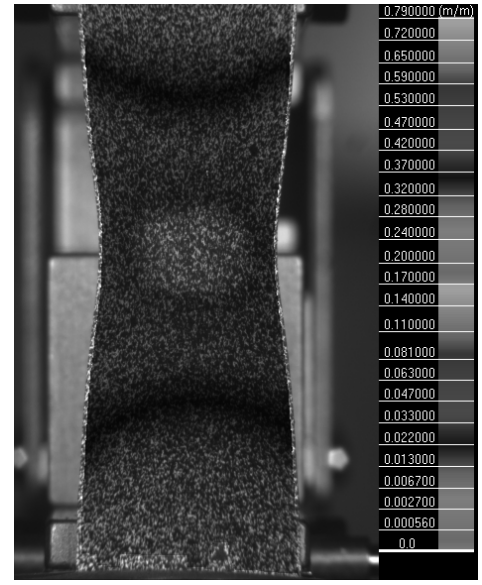

(c)

Figure 6. Equivalent total strain field from image correlation technique postprocessing: a) pre-necking, constant strain. b) onset of necking, strain localization. c) post-necking, large deformation

Figure 7 shows the stress maps computed by applying the procedure presented in the previous section. Again the three contour maps refer to the same time steps of Figure 5 .

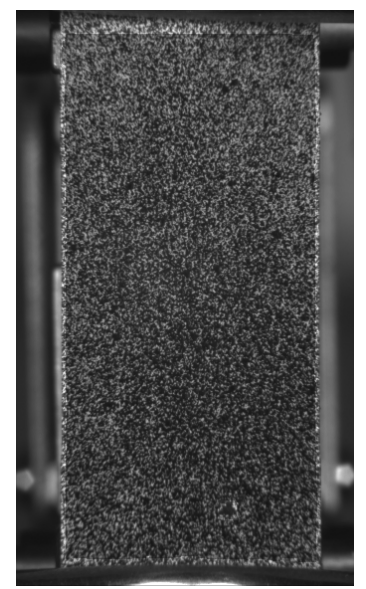

(a)

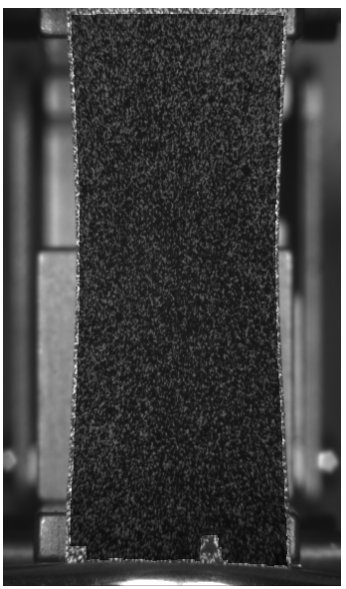

(b)

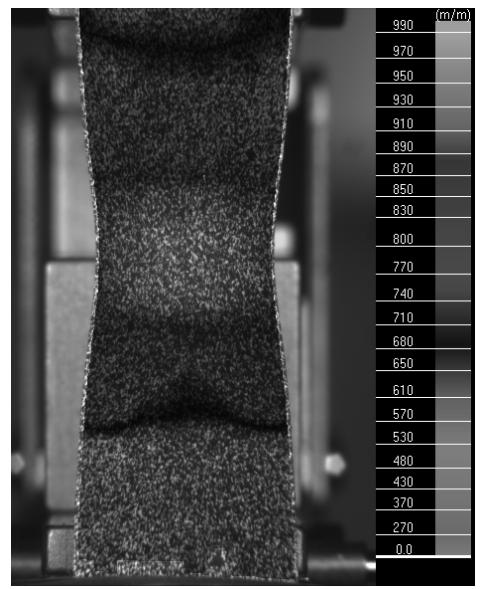

(c)

Figure 7. Von Mises equivalent stress field from the proposed technique postprocessing: a) pre-necking. b) onset of necking. c) post-necking 


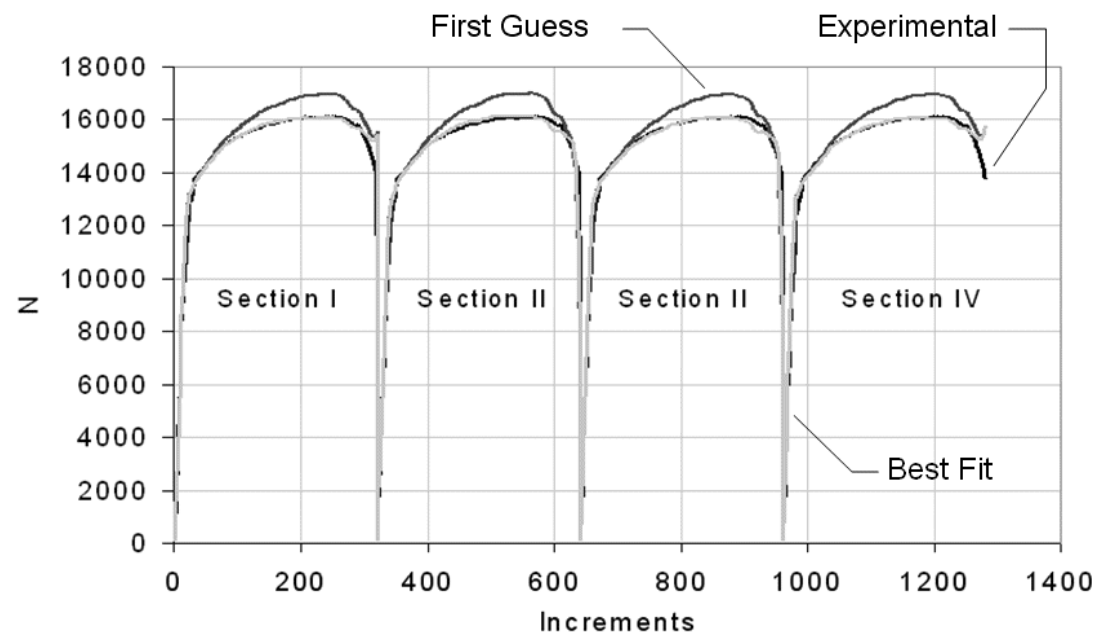

Figure 8. Multi section best fit calibration results. Load-displacement curves. Experimental, first guess and best fit comparison for 4 cross-sections

The calibration procedure described above has been applied with success on the available experimental data. Main results are presented in Figures 8 and 9. In the former, a comparison in terms or global load-displacement curves is reported for 4 equispaced cross-sections selected starting the specimen middle section and $1.25 \mathrm{~mm}$ spaced.

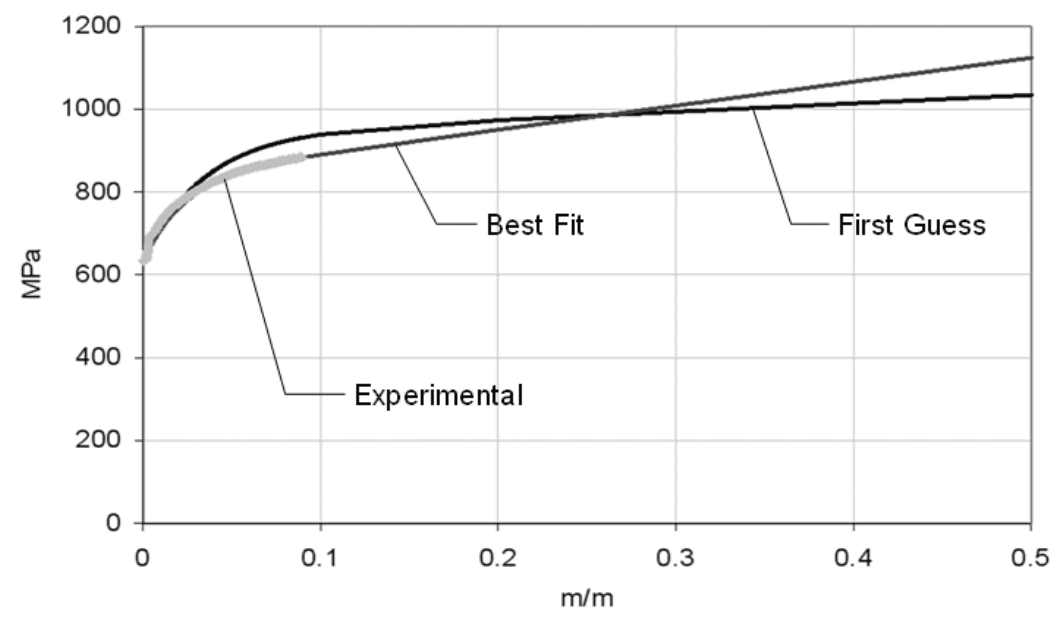

Figure 9. Stress-strain constitutive law. Experimental results up to the onset of necking compared with first guess and optimized curves up to large strains 
This ensures that the region interested by large plastic strains can be "captured". All comparisons are among experimental, first guess and best fit curves. It can be noticed how the response of the proposed procedure closely matches the experimental load curve. In the latter, the final stress-strain curve is reported and can be compared with the experimental one obtained up the onset of necking from a standard tensile test. Finally, Table 1 summarizes the first guess and best fit parameter sets. The $D$ parameter (in this specific application where material curve can be approximated by a straight line in the large strain region) is redundant; therefore, it has been put equal to zero and excluded from the calibration.

Table 1. First guess and best fit parameter sets

\begin{tabular}{cccc}
\hline Parameter & First Guess & Best Fit & \\
\hline$A$ & 300 & 201 & $M P a$ \\
\hline$B$ & 30 & 42.98 & - \\
\hline$C$ & 200 & 578.16 & $M P a$ \\
\hline$D$ & 0 & 0 & $M P a$ \\
\hline
\end{tabular}

To conclude, by means of this method, using digital image correlation, it has been shown that it is possible to extend the knowledge of stress-strain relation far beyond the necking point directly from experimental data. This allows material characterization up to large strain and to correctly model it within FE codes for simulating of all processes where high deformations are expected.

\section{Conclusions}

The proposed method has been successfully implemented in a $\mathrm{C}++$ programming environment. It has been verified on experimental tests performed on flat specimens derived from high-strength steel for thick tube applications. The procedure turned out to be robust and reliable both with the simplex and the non-linear least squares algorithms. In any case, isotropic hardening best fit parameters have been found.

At the very end, the identification of the material constitutive law up to high strains has proven to be practicable, based only on experimental data collected from a standard tensile test on a flat specimen, on condition that the information coming from the image correlation technique are available. These additional data, allowed the elasto-plastic material model characterization by means of local data, unlike the conventional method, where the "tuning" relied on a global data basis.

It must be again pointed out how, for the method to work, plane-stress condition assumption should be strictly verified. This restriction comes from an intrinsic limit 
of the optical technique used, which can obviously investigate material deformation on the specimen surface only.

As future developments, it is worthy to perform supplementary tests on different materials to ensure the procedure applicability on a general material behavior. Then, a validation phase should be accomplished, to confirm the transferability of the identified constitutive law on stress-strain conditions different from those used for calibration. Finally, a comparison with the traditional methods would be valuable too.

\section{References}

Amodio D., Broggiato G. B., Salvini P., "Finite strain analysis by image processing: smoothing techniques", Strain, 31, 1995, p. 151-157.

Amodio D., Broggiato G. B., Campana F., Newaz G. M., "Digital speckle correlation for strain measurement by image analysis", Experimental Mechanics, 43, 2003, p. 396-402.

Besnard G., Hild F., S. Roux S., "Finite-Element Displacement Fields Analysis from Digital Images: Application to Portevin-Le Châtelier Bands", Experimental Mechanics, 46, 2006, p. $789-803$.

Bridgman P. W., Studies in large plastic flow and fracture, McGraw-Hill, New York, 1952.

Broggiato G. B., "Adaptive image correlation technique for full-field strain measurement", Proceedings of ICEM12 - $12^{\text {th }}$ International Conference on Experimental Mechanics, Bari, Italy, 2004.

Broggiato G. B., Casarotto L., Del Prete Z., Maccarrone D., "Full-field strain rate measurement by white-light speckle image correlation", Strain, published online (early view), DOI: 10.1111/j.1475-1305.2008.00446.x2008, 2008.

Bruck H. A., McNeill S. R., Sutton M. A., Peters W.H., "Digital image correlation using Newton-Raphson method of partial differential correction", Experimental Mechanics, 29, 1989, p. 261-267.

Cheng P., Sutton M. A., Schreier H. W., McNeill, S. R., "Full-field speckle pattern image correlation with B-spline deformation function", Experimental Mechanics, 42, 2002, p. 344-352.

Chung T. J., Continuum Mechanics, Prentice-Hall International Edition, 1988.

Cooreman S., Lecompte D., Sol H., Vantomme J., Debruyne D., "Elasto-plastic material parameter identification by inverse methods: Calculation of the sensitivity matrix". International Journal of Solids and Structures, 44, 2007, p. 4329-4341.

Grediac M., Pierron, F., "Applying the Virtual Fields Method to the identification of elastoplastic constitutive parameters", International Journal of Plasticity, 22, 2002, p. 602-627.

Khan A. S., Huang S., Continuum Theory of Plasticity, John Wiley \& Sons, 1995.

Ling Y., "Uniaxial True Stress-Strain after Necking”, AMP Journal of Technology, 5, 1996, p. $37-48$. 
McClintock F. A., Argon A. S., Mechanical behavior of materials, Addison-Wesley Publishing Company, 1966, p. 322-324.

Robert L., Orteu J. J., "Assessment of digital image correlation performances", Proceedings of ICEM13-13 ${ }^{\text {th }}$ International Conference on Experimental Mechanics, Alexandroupolis, Greece, 2007.

Sutton M. A., Wolters W. J., Peters W. H., Ranson W. F., McNeill S. R., "Determination of displacements using an improved digital correlation method", Image and Vision Computing., vol. 1, $\mathrm{n}^{\circ} 3$, 1983, p. 133-139. 